\title{
Synthesis and Characterization of Hydrolysis Grown Zinc Oxide Nanorods
}

\author{
Arun Vasudevan, Soyoun Jung, and Taeksoo Ji \\ Department of Electrical Engineering, University of Arkansas, Fayetteville, AR 72701, USA \\ Correspondence should be addressed to Taeksoo Ji, tji@uark.edu
}

Received 23 June 2011; Accepted 14 July 2011

Academic Editors: M. Huang, D. Losic, A. Taubert, and D. K. Yi

Copyright () 2011 Arun Vasudevan et al. This is an open access article distributed under the Creative Commons Attribution License, which permits unrestricted use, distribution, and reproduction in any medium, provided the original work is properly cited.

We present $\mathrm{ZnO}$ nanorods grown by a low-cost hydrolysis method with a rod diameter on the order of $30-40 \mathrm{~nm}$ and spacing on the order of $20-40 \mathrm{~nm}$ that find their applications in the field of solar cells and UV photodetectors with high efficiency and sensitivity. The effect of different process parameters on nanorod growth, such as the seed layer orientation in the growth medium, and the concentration of the growth medium were studied while characterizing the structure of the grown nanorods using XRD, EDAX, and SEM.

\section{Introduction}

Zinc oxide $(\mathrm{ZnO})$ is a II-VI semiconductor with a high band gap of $3.3 \mathrm{eV}$ and binding energy of $60 \mathrm{meV}$ [1]. These values are much higher when compared to other semiconductor materials such as $\mathrm{ZnSe}(22 \mathrm{meV}), \mathrm{ZnS}(40 \mathrm{meV})$, and $\mathrm{GaN}$ $(25 \mathrm{meV})$ which makes $\mathrm{ZnO}$ a better candidate for room temperature UV laser fabrication [2]. Since $\mathrm{ZnO}$ has a wurtzite structure lacking any center of symmetry and a large electrochemical coupling resulting in strong piezoelectric and pyroelectric properties, it can be employed for applications in mechanical actuators and piezoelectric sensors [3]. In the form of thin films, $\mathrm{ZnO}$ is also a very promising alternative in flat display screens [4-6] for tin-doped indium oxides (ITO) for which there is a limited natural source.

Intensive research has been focused on fabricating onedimensional $\mathrm{ZnO}$ nanostructures and on correlating their morphologies with their size-related optical and electrical properties [7-10]. Even though various kinds of $\mathrm{ZnO}$ nanostructures have been realized, such as nanodots, nanorods, nanowires, nanobelts, nanotubes, nanobridges, nanonails, nanowalls, nanohelixes, seamless nanorings, mesoporous single-crystal nanowires, and polyhedral cages [3, 11, 12], $\mathrm{ZnO}$ nanorods (NRs) and nanowires (NWs) have been the most widely studied because of their easy formation and device applications. They can be used as both interconnects and functional units in fabricating electronic, optoelectronic, electrochemical, and electromechanical nanodevices [13, 14].

Different approaches have been adopted for $\mathrm{ZnO}$ growth such as vapor phase transport technique [15], thermal decomposition of precursors [16], oxidation of zinc metal [17], metalorganic vapor phase [18]. These techniques, however, require moderate to high temperature. For example, the evaporation technique for $\mathrm{ZnO} \mathrm{NRs}$ growth requires 800 $900^{\circ} \mathrm{C}[19,20]$. Even though the MOCVD technique using organometallic zinc precursors brings down the growth temperature to $450^{\circ} \mathrm{C}$ [21], the growth of NRs on commercial scale using these techniques is expensive due to costly insulating substrates needed for the oriented growth and cost associated with vapor deposition system. In comparison, the growth of $\mathrm{ZnO} \mathrm{NRs}$ based on a hydrolysis process is much cheaper because this method does not require sophisticated deposition systems or stringent experimental conditions, and also cheaper substrates such as glass or plastic can be used for the growth of well-oriented $\mathrm{ZnO}$ NRs. In solution growth, since a seed layer is used, the growth of NRs takes place through site-specific nucleation, thus facilitating the manipulation of the density and orientation of NRs.

One of the major applications of $\mathrm{ZnO}$ NRs prepared using low-temperature hydrolysis synthesis lies in the fabrication of low-cost, efficient hybrid solar cells. Organic 
solar cells can be fabricated using simple solution processing techniques that provide the possible option for low-cost photovoltaic devices, but their efficiency is very low [2226]. Recently, a hybrid solar cell that combines organic bulk heterojunction solar cell with vertical NRs of inorganic semiconductors has been envisioned to improve the charge transport in the bulk heterojunction, leading to high efficiency [27]. These vertical NRs with their high mobility and electron acceptance provide better charge transport to the electrodes and also better charge separation that occurs at inorganic-organic interface in addition to the charge separation taking place at an organic-organic interface. Though the charge separation at inorganic-organic interface is not more efficient than that at organic-organic interface [28], it can be improved if the spacing between the NRs is of the carrier diffusion length of the organic semiconductors, that is, 10-20 nm [29]. The high electron mobility and easy low-temperature synthesis make ZnO NRs an excellent material for use in bulk heterojunction organic solar cells to serve as the transport of the carriers [27].

Another $\mathrm{ZnO}$ NRs application that has received recent attention is for UV photodetectors utilizing their good UV response. The high UV response of $\mathrm{ZnO}$ is attributed to its wide bandgap, large surface-area-to-volume ratio, and high internal photoconductivity gain stemming from the surface enhanced electron-hole separation efficiency [30]. While easily integrated with portable micro/nanosystems, $\mathrm{ZnO}$ based UV photodetectors with intrinsic "visible blindness" (the bandgap energy of $\mathrm{ZnO}$ being higher than visible light energy, the response of the detector to visible light is nil) can be operated at high temperature and in harsh environments [30]. The sensitivity of $\mathrm{ZnO}$ photodetector increases with decrease in the rod diameter of the $\mathrm{ZnO}$ NRs [31]. This is because the UV received by the detector is proportional to the surface area while the UV effect is inversely proportional to the volume (excited electron density). Thus, the thinner the NR, the more the sensitivity.

In this paper, we discuss the growth of $\mathrm{ZnO}$ NRs with diameters of $30-40 \mathrm{~nm}$ where the spacing between the rods is equivalent to the carrier diffusion length of organic semiconductors using a facile and the low-cost hydrolysis method prepared from zinc nitrate hexahydrate and hexamethylenetetramine. Rods of these dimensions are required to improve the efficiency of organic solar cells and sensitivity of UV detector. Dependence of the properties of the grown $\mathrm{ZnO}$ NRs on the orientation of the seed layer and the concentration of the growth medium is also presented.

\section{Experimental}

2.1. Materials. For the growth of $\mathrm{ZnO}$ NRs, a two-step hydrolysis process was employed. The first step was to deposit $\mathrm{ZnO}$ thin films which were subsequently used as seed layers for the aqueous solution growth of $\mathrm{ZnO}$ NRs. Zinc acetate $\left.\left(\mathrm{Zn}\left(\mathrm{CH}_{3} \mathrm{COO}\right)\right)_{2}, 99.98 \%\right)$, ethanolamine $\left(\mathrm{HOCH}_{2} \mathrm{CH}_{2}\right.$ $\left.\mathrm{NH}_{2}, 99 \%\right)$, and ethanol (99.5\%) purchased from Alfa Aesar and Sigma-Aldrich, respectively, were used to form the seed layer. Zinc nitrate hexahydrate $\left(\mathrm{Zn}\left(\mathrm{NO}_{3}\right)_{2} 6 \mathrm{H}_{2} \mathrm{O}\right.$,
99-100\%) and hexamethylenetetramine $\left(\left(\mathrm{CH}_{2}\right)_{6} \mathrm{~N}_{4}, 99 \%\right)$ were purchased from J. T. Baker to grow the $\mathrm{ZnO}$ NRs. The whole device was fabricated on quartz substrate having dimensions $25 \times 25 \times 0.7 \mathrm{~mm}$. Prior to the deposition of seed layers, the quartz substrates were cleaned ultrasonically in detergent solution, acetone, isopropyl alcohol, and distilled water sequentially.

2.2. Preparation of the Seed Layer. First, the seed layer solution was prepared by dissolving zinc acetate and ethanolamine in the ratio of $1: 1$ in ethanol and stirring it for $1 \mathrm{hr}$ at $70^{\circ} \mathrm{C}$. For the sake of comparison, different values of solution concentration (from $0.01 \mathrm{M}$ to $0.1 \mathrm{M}$ ) were tried. It was observed that a film of the seed layer with uniform thickness was obtained with a $0.1 \mathrm{M}$ concentration of zinc acetate and ethanolamine solution. In order to further optimize the ratio of zinc acetate and ethanolamine, the concentration of ethanolamine was varied from $0.05 \mathrm{M}$ to $0.35 \mathrm{M}$ while keeping the concentration of zinc acetate value unchanged at $0.1 \mathrm{M}$. The solution was then spun onto a substrate at $1000 \mathrm{RPM}$ for $20 \mathrm{~s}$, followed by annealing at $350^{\circ} \mathrm{C}$ for $1 \mathrm{hr}$ to form a $\mathrm{ZnO}$ thin film seed layer.

2.3. Preparation of the $\mathrm{ZnO}$ Nanorods. The growth of $\mathrm{ZnO}$ NRs was conducted by suspending the as-prepared substrates with the seed layer in a solution prepared from zinc nitrate and hexamethylenetetramine. Both zinc nitrate and hexamethylenetetramine were dissolved in distilled water by stirring it for 2 hour at room temperature. The seed layer was then immersed in the solution, aged in an oven at $90^{\circ} \mathrm{C}$ for 2 hours, and carefully rinsed by distilled water for several times. A comparative investigation was also conducted on the effect of the seed layer orientation in the NR solution on the growth of the NRs. While changing the concentration of hexamethylenetetramine in the solution, the zinc nitrate was kept constant at $0.025 \mathrm{M}$. The samples were characterized structurally using Rigaku X-ray diffractometer with $\mathrm{CuK}_{\alpha}$ radiation of wavelength $1.541874 \AA$. For morphology and composition analysis Philips, XL30 scanning electron microscope was used.

\section{Results and Discussion}

3.1. Surface Morphology of the Seed Layer. The SEM images on the surface of the spin-coated seed layer for different ethanolamine concentration with a fixed concentration of zinc acetate at $0.1 \mathrm{M}(0.05,0.1,0.15,0.2,0.25$, and $0.3 \mathrm{M})$ are shown in Figure 1. It was observed that a film of uniform thickness was obtained with a concentration of the ethanolamine at $0.05 \mathrm{M}$ and $0.1 \mathrm{M}$ as shown in Figures 1(a) and $1(\mathrm{~b})$ while the rest of the films seemed nonuniform in thickness as shown in Figures 1(c) to 1(f). In addition, when the concentration of zinc acetate and ethanolamine went below $0.1 \mathrm{M}$ holding the ratio at $1: 1$, it appeared that the surface of thin films became neither uniform nor smooth similar to the SEM image shown in Figure 1(c), where the white spot droplets were ascribed to the accumulation of zinc acetate during spin coating. 


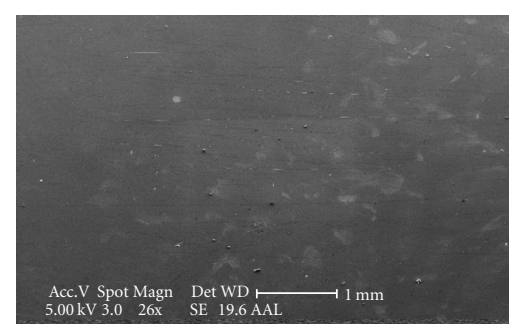

(a)

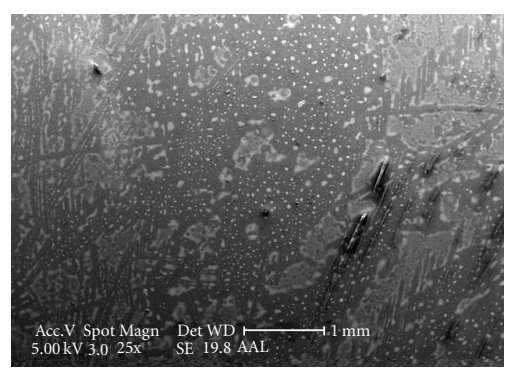

(d)

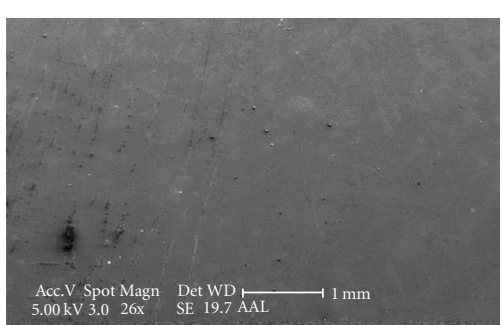

(b)

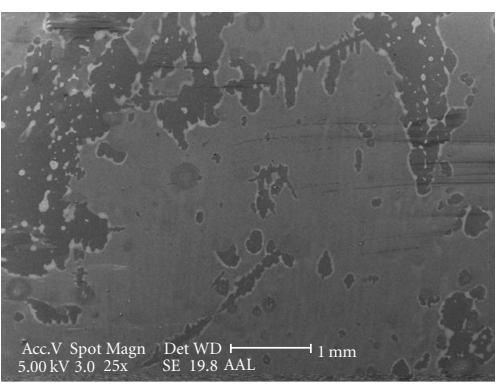

(e)

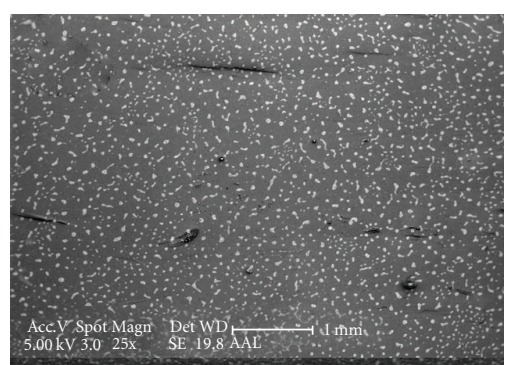

(c)

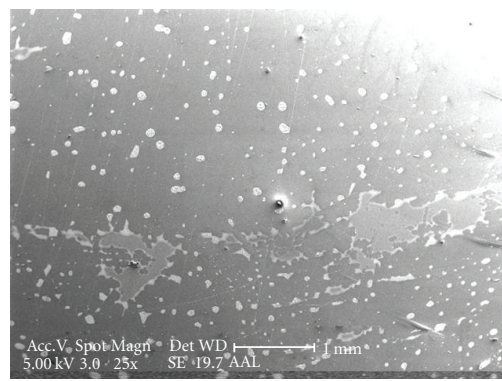

(f)

FIGURE 1: SEM image of the spin-coated seed layer for different ethanolamine concentration with a fixed concentration of zinc acetate at 0.1 M: (a) 0.05 M, (b) $0.1 \mathrm{M}$, (c) $0.15 \mathrm{M}$, (d) $0.2 \mathrm{M}$, (e) $0.25 \mathrm{M}$, and (f) $0.3 \mathrm{M}$.

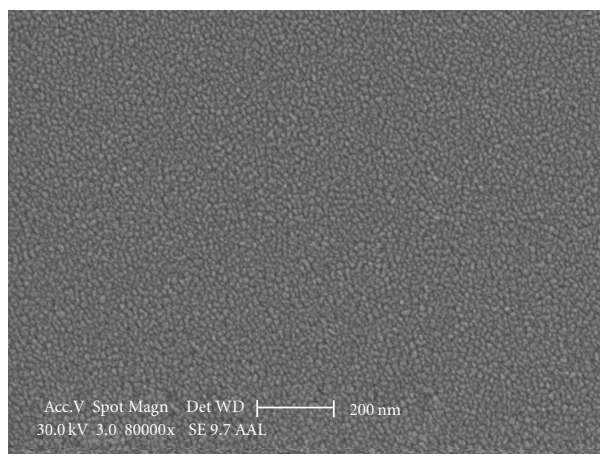

(a)

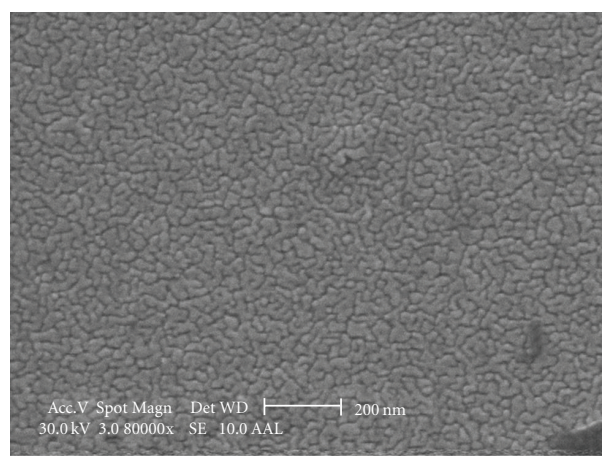

(b)

Figure 2: SEM image of the seed layer. (a) Prepared after storing the solution for one day. (b) After storing the solution for one month.

Figure 2 compares the surface morphologies of the $\mathrm{ZnO}$ thin films spin coated from the same $0.1 \mathrm{M}$ solution of zinc acetate and ethanolamine but for different storage times. It is easily noted that the diameter of the seeds spin-coated the next day after the preparation of the solution (Figure 2(a)) is much smaller than that of the seeds deposited onto a substrate after storing the solution for one month (Figure 2(b)). This increase in size may stem from coalescence of the seeds leading to the formation of larger seeds in solution. Thus, along with the optical concentration of ethanolamine between $0.05 \mathrm{M}$ and $0.1 \mathrm{M}$ that results in a uniform seed film as shown in Figure 1, it appears that the short storing time of solution after synthesizing prevent the seed from becoming larger, both of which help generate NRs with sizes close to the Debye length [32].

\subsection{Characterization of $\mathrm{ZnO}$ Nanorods}

3.2.1. SEM Characterization. ZnO NRs were grown with the seed layer suspended horizontally or vertically in the growth solution. The concentration of the growth medium was varied with different concentration of hexamethylenetetramine, HMT $(0.015 \mathrm{M}, 0.02 \mathrm{M}, 0.025 \mathrm{M}, 0.03 \mathrm{M}, 0.035 \mathrm{M}$, and $0.055 \mathrm{M}$ ), while the zinc nitrate hexahydrate concentration remained same at $0.025 \mathrm{M}$. It was observed that the rods were grown with diameters of $30-40 \mathrm{~nm}$, spacing of 20 $40 \mathrm{~nm}$, and length of $0.5 \mu \mathrm{m}$. In addition, it was noted that the $\mathrm{ZnO}$ NRs were hexagonally shaped for all the varied concentrations of the HMT no matter how the seed layer is oriented in the solution. Figures 3(a) and 3(b) show the SEM image of the NRs for HMT concentration of $0.015 \mathrm{M}$ for both 


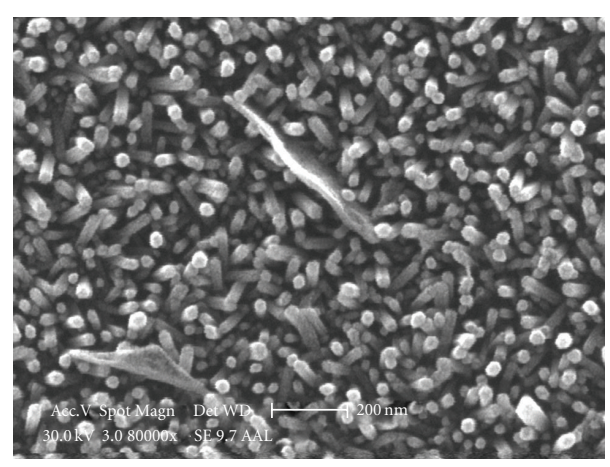

(a)

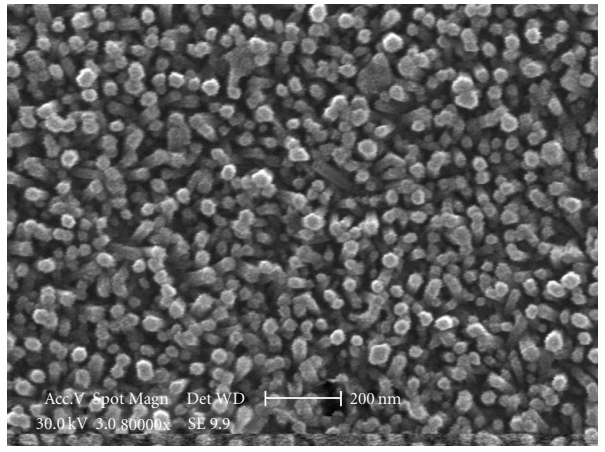

(b)

FIGURE 3: SEM images of the ZnO NRs grown with concentration of hexamethylenetetramine at $0.015 \mathrm{M}$ and zinc nitrate hexahydrate at 0.025 M. (a) Horizontal orientation of the seed layer. (b) Vertical orientation of the seed layer.

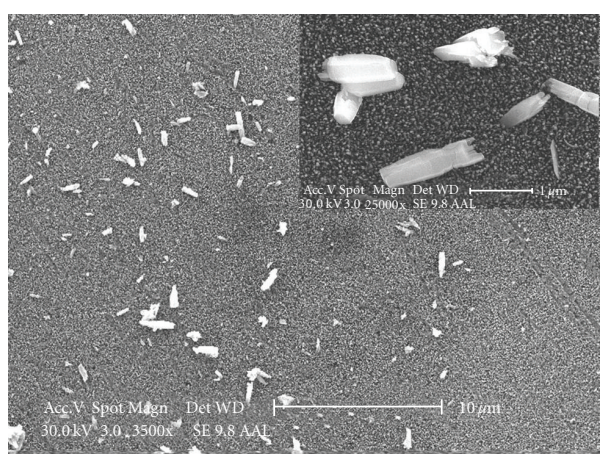

(a)

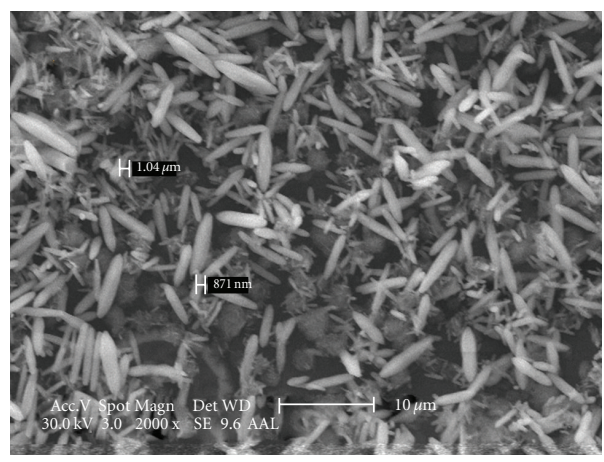

(b)

Figure 4: SEM image of the ZnO particles that settles on the surface of ZnO's nanorod film from the growth solution (a) $0.055 \mathrm{M}$ of HMT and (b) $0.015 \mathrm{M}$ of HMT. Inset in (a) shows the magnified image of the $\mathrm{ZnO}$ particles on the surface of the $\mathrm{ZnO}$ nanorod film.

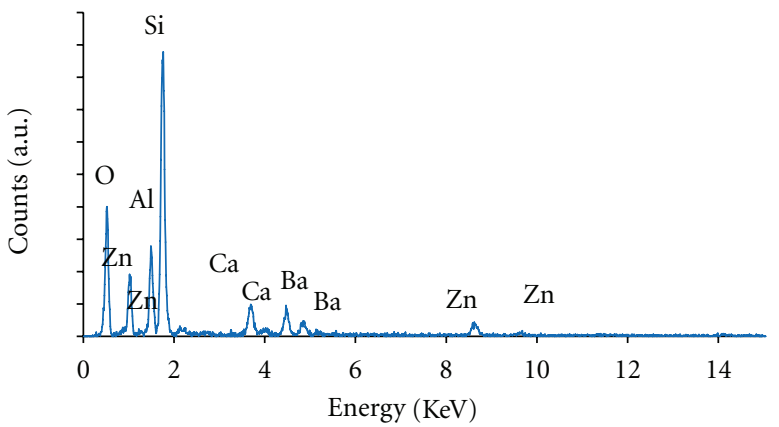

FIGURE 5: EDAX spectrum of the ZnO NRs on vertical orientation of seed layer grown with concentration of HMT $0.015 \mathrm{M}$ and zinc nitrate hexahydrate at $0.025 \mathrm{M}$.

horizontal and vertical orientation, respectively, which look similar to the NRs images of the other HMT concentrations and orientations (not shown here).

When the molar concentration of the HMT is less than that of the zinc nitrate hexahydrate, there should be a decrease in the growth rate of $\mathrm{ZnO} \mathrm{NRs}$ due to deficiency in oxygen, in turn, affecting the length of the NRs. However, the SEM images show that the length of the rod is not much

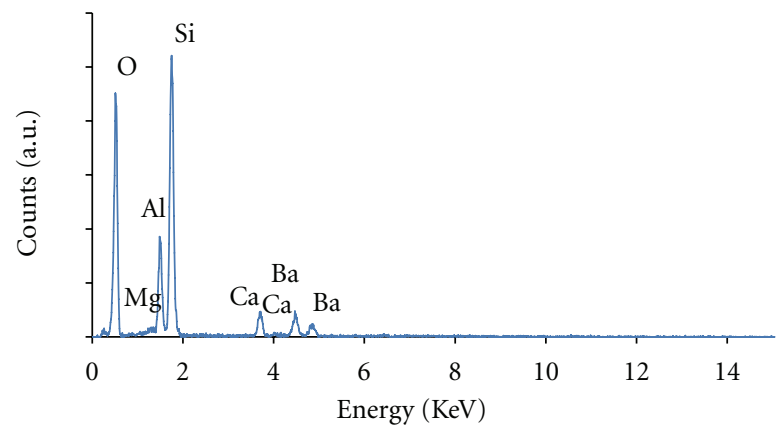

Figure 6: EDAX spectrum of the substrate.

affected even when the concentration of HMT was below the molar concentration of zinc nitrate hexahydrate. This suggests that only a part of the existing zinc ions was used up for the NRs growth.

One of the disadvantages of orienting the seed layer horizontally in the growth solution is that, in addition to the growth of $\mathrm{ZnO} \mathrm{NRs}, \mathrm{ZnO}$ particles with cylindrical and spindle shape are also formed in the growth solution, which due to gravity will settle on the growing $\mathrm{ZnO}$ NRs. The $\mathrm{ZnO}$ particles exhibit a spindle shape when the concentration 


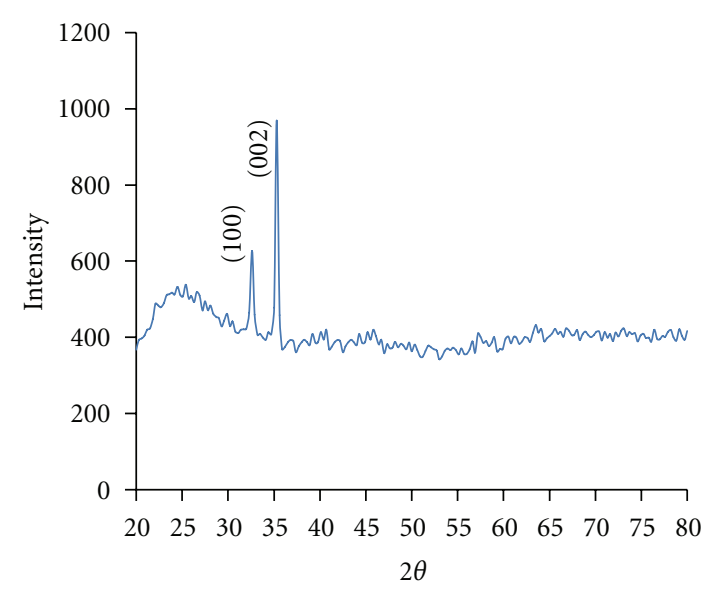

(a)

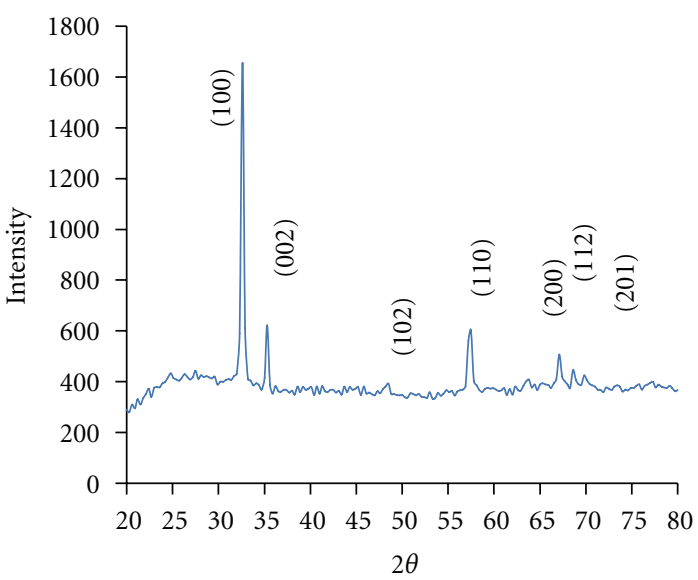

(c)

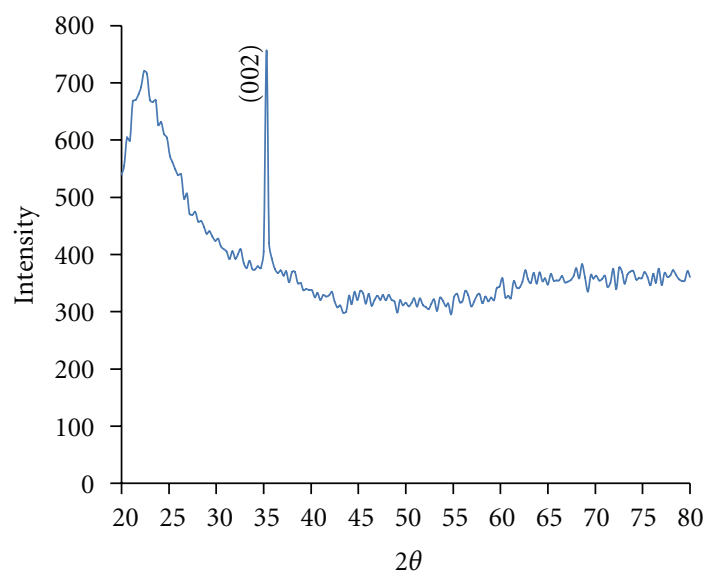

(e)

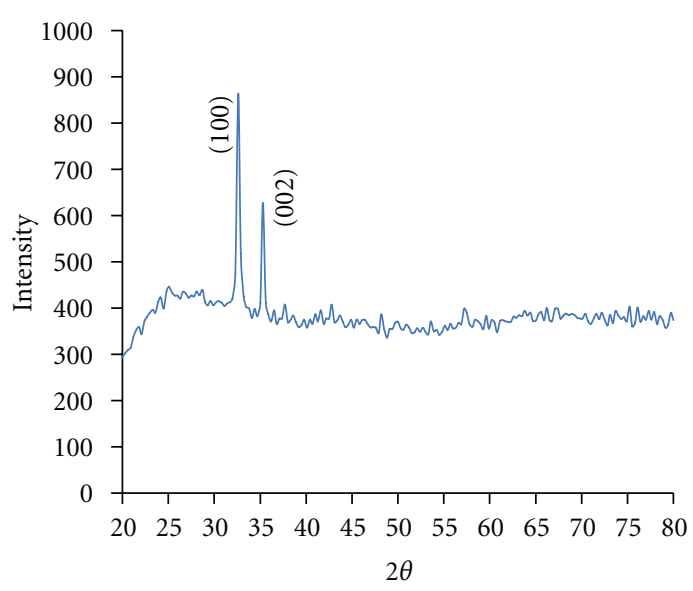

(b)

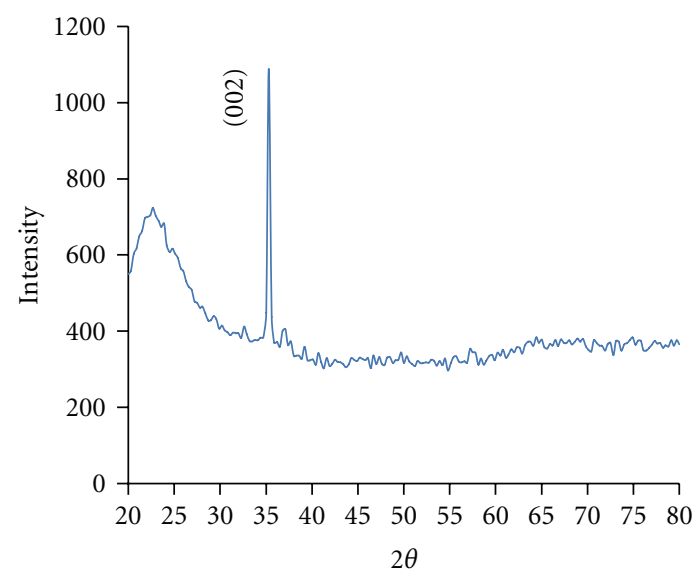

(d)

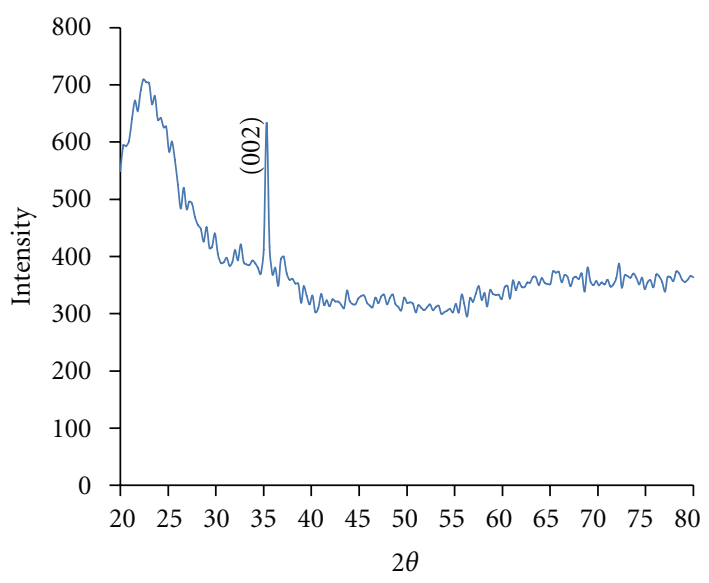

(f)

FIGURE 7: XRD patterns of ZnO NRs grown with the seed layer oriented horizontally and vertically for different HMT concentration and zinc acetate at constant molar concentration of $0.025 \mathrm{M}$. Horizontal orientation (a) $0.035 \mathrm{M}$, (b) $0.025 \mathrm{M}$, and (c) $0.015 \mathrm{M}$, and vertical orientation (d) $0.035 \mathrm{M}$, (e) $0.025 \mathrm{M}$, and (f) $0.015 \mathrm{M}$.

of HMT is $0.015 \mathrm{M}$ as shown in Figure 4 (b), while for the other concentration the particles are cylindrical in shape. Figure 4(a), and the inset shows the particles with cylindrical shape settled on the surface when the concentration of the HMT is $0.055 \mathrm{M}$.
3.2.2. EDAX Spectrum. Figure 5 shows the EDAX spectrum for the $\mathrm{ZnO}$ NRs whose seed layers were oriented vertically in the growth medium with ethanolamine concentration of $0.015 \mathrm{M}$ and zinc nitrate hexahydrate of $0.025 \mathrm{M}$, which is similar to that of NRs on the horizontal orientation of seed 
layer. For both the horizontal and vertical orientation, it turned out that even changing the concentration of HMT does not affect the intensity of the spectrum. The EDAX spectrum also confirms the rods grown are $\mathrm{ZnO}$ with the zinc and oxygen combined in the ratio $1: 1.04$. The other peaks visible in the spectrum are due to the substrate itself and impurities in the substrate (Figure 6).

3.2.3. Structural Characterization. The orientation of the crystal planes of the ZnO NRs can be determined from XRD. The XRD pattern obtained can be matched to the standard data for ZnO (ICDD no. 00-036-1451). Figure 7 shows the XRD pattern of the NRs when the seed layer is suspended in different HMT concentration of solutions horizontally (Figures $7(\mathrm{a})-7(\mathrm{c})$ ) and vertically (Figures $7(\mathrm{~d})-7(\mathrm{f})$ ). For horizontal orientation of the seed layer, the $\mathrm{ZnO}$ NRs with concentration of HMT of $0.055 \mathrm{M}$ and $0.035 \mathrm{M}$ exhibited the XRD patterns similar to shown in Figure 7(a). The two peaks in the spectrum correspond to (100) and (002) planes where the (002) planes represents the planes perpendicular to the $c$-axis. The peaks corresponding to (100) planes in the spectrum come from the $\mathrm{ZnO}$ crystals that crystallize from the solution and get settled on the surface of the rods. The SEM image of the $\mathrm{ZnO}$ crystals on the surface is shown in Figure 4(a). It was observed that when the HMT concentration was decreased to $0.03 \mathrm{M}$, the intensity of the (002) planes weakened while the intensity of (100) planes increased as shown in Figure 7(b). Similar spectrum was obtained when the concentration was further decreased to $0.025 \mathrm{M}$. The increase in intensity of the (100) plane suggests that the amount of $\mathrm{ZnO}$ crystals that settle on the surface increases. The reason for the decrease in intensity of the (002) planes can be understood from the XRD pattern for the vertical orientation of the seed layer as explained below. As shown in Figure 7(c), with further decrease in concentration of the HMT $(0.02 \mathrm{M}$ and $0.015 \mathrm{M})$, the intensity of $(002)$ plane decreases slightly while the intensity of the (100) planes increases. As explained above the increase in (100) plane is likely due to the $\mathrm{ZnO}$ crystals on the surface. The increase in intensity of the (100) planes with decrease in HMT concentration shows that crystallization of the $\mathrm{ZnO}$ crystals from the solution is enhanced on decreasing the HMT concentration.

Figures 7(d), 7(e), and 7(f) show the XRD patterns of the $\mathrm{ZnO} \mathrm{NRs}$ for vertical orientation of the seed layer grown with HMT of $0.035 \mathrm{M}, 0.025 \mathrm{M}$, and $0.015 \mathrm{M}$, respectively. It is easily noted that they exhibit only the (002) peak whose intensity decreases with decreasing concentration of the HMT. The absence of the peaks other than (002) planes shows that by orienting the seed layer vertically we can avoid the $\mathrm{ZnO}$ crystals from getting deposited on the surface. The reason for the decrease in intensity of (002) plane can be either due to random alignment of the rods or decrease in crystallinity of the rods. Since there are no peaks other than the (002) plane, the decrease in intensity of the rod due to orientation can be ruled out. The length of the rods for all the samples is the same, determined from SEM, and the density and composition of the rods are also the same because of the identical EDAX spectrum. Then the most probable reason for the decrease in intensity is ascribed to the decrease in crystallinity of $\mathrm{ZnO}$ NRs. The role of HMT during the growth of $\mathrm{ZnO}$ rods is to supply oxygen ions that combine with the zinc ions from zinc nitrate hexahydrate to form $\mathrm{ZnO}$. Thus, it appears that low HMT concentration leads to the lack of enough oxygen ions giving rise to deteriorating $\mathrm{ZnO}$ crystallinity.

\section{Summary}

Comparison studies have been performed to optimize the growth of $\mathrm{ZnO} \mathrm{NRs}$ based on a two-process, low-cost hydrolysis method. It turns out that the vertical orientation of the seed layer in the growth medium results in better characteristics of $\mathrm{ZnO}$ NRs when compared to the horizontal orientation in terms of morphology, surface cleanness, crystallinity, $\mathrm{ZnO}$ orientation and so forth. It is found that a uniform $\mathrm{ZnO}$ layer with small sizes of seeds can be obtained with a concentration of zinc acetate at $0.1 \mathrm{M}$ and ethanolamine at $0.05 \mathrm{M}$ or $0.1 \mathrm{M}$. It is also observed that, for the growth of $\mathrm{ZnO}$ NRs the concentration of HMT higher than $0.035 \mathrm{M}$ at $0.025 \mathrm{M}$ of zinc nitrate hexahydrate creates good crystalline and vertical orientation of NRs. Since the distance between adjacent NRs varies from 20 to $40 \mathrm{~nm}$, which is close to the diffusion length of the carriers of organic materials, these $\mathrm{ZnO}$ NRs can be suitable candidates for hybrid solar cell applications. In addition, the narrow diameter of the NRs grown $(30-40 \mathrm{~nm})$ in this work can find applications in UV photodetectors with high sensitiveness.

\section{Acknowledgment}

This work was partially supported by a grant from the Arkansas Biosciences Institute.

\section{References}

[1] D. G. Thomas, "The exciton spectrum of zinc oxide," Journal of Physics and Chemistry of Solids, vol. 15, no. 1-2, pp. 86-96, 1960.

[2] M. H. Huang, S. Mao, H. Feick et al., "Room-temperature ultraviolet nanowire nanolasers," Science, vol. 292, no. 5523, pp. 1897-1899, 2001.

[3] Z. L. Wang, "Nanostructures of zinc oxide," Materials Today, vol. 7, no. 6, pp. 26-33, 2004.

[4] D. S. Ginley and C. Bright, "Transparent conducting oxides," MRS Bulletin, vol. 25, no. 8, pp. 15-21, 2000.

[5] B. G. Lewis and D. C. Paine, "Applications and processing of transparent conducting oxides," MRS Bulletin, vol. 25, no. 8, pp. 22-27, 2000.

[6] A. J. Freeman, K. R. Poeppelmeier, T. O. Mason, R. P. H. Chang, and T. J. Marks, "Chemical and thin-film strategies for new transparent conducting oxides," MRS Bulletin, vol. 25, no. 8, pp. 45-51, 2000.

[7] K. Keem, H. Kim, G.-T. Kim et al., "Photocurrent in $\mathrm{ZnO}$ nanowires grown from Au electrodes," Applied Physics Letters, vol. 84, no. 22, pp. 4376-4378, 2004.

[8] M. S. Arnold, P. Avouris, Z. W. Pan, and Z. L. Wang, "Field-effect transistors based on single semiconducting oxide nanobelts," Journal of Physical Chemistry B, vol. 107, no. 3, pp. 659-663, 2003. 
[9] C. J. Lee, T. J. Lee, S. C. Lyu, Y. Zhang, H. Ruh, and H. J. Lee, "Field emission from well-aligned zinc oxide nanowires grown at low temperature," Applied Physics Letters, vol. 81, no. 19, Article ID 3648, 3 pages, 2002.

[10] W. I. Park, Y. H. Jun, S. W. Jung, and G.-C. Yi, "Excitonic emissions observed in $\mathrm{ZnO}$ single crystal nanorods," Applied Physics Letters, vol. 82, no. 6, pp. 964-966, 2003.

[11] J.-H. Park, H.-J. Choi, Y.-J. Choi, S.-H. Sohn, and J.-G. Park, "Ultrawide ZnO nanosheets," Journal of Materials Chemistry, vol. 14, no. 1, pp. 35-36, 2004.

[12] J. H. Park, H. J. Choi, and J. G. Park, "Scaffolding and filling process: a new type of 2D crystal growth," Journal of Crystal Growth, vol. 263, no. 1-4, pp. 237-242, 2004.

[13] Z. L. Wang, "Characterizing the structure and properties of individual wire-like nanoentities," Advanced Materials, vol. 12, no. 17 , pp. 1295-1298, 2000.

[14] J. Hu, T. W. Odom, and C. M. Lieber, "Chemistry and physics in one dimension: synthesis and properties of nanowires and nanotubes," Accounts of Chemical Research, vol. 32, no. 5, pp. 435-445, 1999.

[15] M. H. Huang, Y. Wu, H. Feick, N. Tran, E. Weber, and P. Yang, "Catalytic growth of zinc oxide nanowires by vapor transport," Advanced Materials, vol. 13, no. 2, pp. 113-116, 2001.

[16] C. Xu, G. Xu, Y. Liu, and G. Wang, "A simple and novel route for the preparation of $\mathrm{ZnO}$ nanorods," Solid State Communications, vol. 122, no. 3-4, pp. 175-179, 2002.

[17] Y. W. Wang, L. D. Zhang, G. Z. Wang, X. S. Peng, Z. Q. Chu, and C. H. Liang, "Catalytic growth of semiconducting zinc oxide nanowires and their photoluminescence properties," Journal of Crystal Growth, vol. 234, no. 1, pp. 171-175, 2002.

[18] W. I. Park, D. H. Kim, S.-W. Jung, and G.-C. Yi, "Metalorganic vapor-phase epitaxial growth of vertically well-aligned $\mathrm{ZnO}$ nanorods," Applied Physics Letters, vol. 80, no. 22, pp. 4232 4234, 2002.

[19] P. Yang, H. Yan, S. Mao et al., "Controlled growth of $\mathrm{ZnO}$ nanowires and their optical properties," Advanced Functional Materials, vol. 12, no. 5, pp. 323-331, 2002.

[20] B. D. Yao, Y. F. Chan, and N. Wang, "Formation of $\mathrm{ZnO}$ nanostructures by a simple way of thermal evaporation," Applied Physics Letters, vol. 81, no. 4, pp. 757-759, 2002.

[21] W. I. Park, G. C. Yi, M. Kim, and S. J. Pennycook, "ZnO nanoneedles grown vertically on Si substrates by non-catalytic vapor-phase epitaxy," Advanced Materials, vol. 14, no. 24, pp. 1841-1843, 2002.

[22] A. M. Peiró, P. Ravirajan, K. Govender et al., "Hybrid polymer/metal oxide solar cells based on $\mathrm{ZnO}$ columnar structures," Journal of Materials Chemistry, vol. 16, no. 21, pp. 2088-2096, 2006.

[23] A. Baumann, J. Lorrmann, C. Deibel, and V. Dyakonov, "Bipolar charge transport in poly(3-hexyl thiophene)/methanofullerene blends: a ratio dependent study," Applied Physics Letters, vol. 93, no. 25, Article ID 252104, 2008.

[24] F. C. Krebs, "Fabrication and processing of polymer solar cells: a review of printing and coating techniques," Solar Energy Materials and Solar Cells, vol. 93, no. 4, pp. 394-412, 2009.

[25] J. Bouclé, P. Ravirajan, and J. Nelson, "Hybrid polymer-metal oxide thin films for photovoltaic applications," Journal of Materials Chemistry, vol. 17, no. 30, pp. 3141-3153, 2007.

[26] T. Kuwabara, H. Sugiyama, T. Yamaguchi, and K. Takahashi, "Inverted type bulk-heterojunction organic solar cell using electrodeposited titanium oxide thin films as electron collector electrode," Thin Solid Films, vol. 517, no. 13, pp. 3766-3769, 2009.
[27] K. Takanezawa, K. Hirota, Q.-S. Wei, K. Tajima, and K. Hashimoto, "Efficient charge collection with $\mathrm{ZnO}$ nanorod array in hybrid photovoltaic devices," Journal of Physical Chemistry C, vol. 111, no. 19, pp. 7218-7223, 2007.

[28] K. Takanezawa, K. Tajima, and K. Hashimoto, "Charge separation interfaces in polymer photovoltaic devices hybridized with $\mathrm{ZnO}$ nanorod arrays," Japanese Journal of Applied Physics, vol. 47, no. 10, pp. 8049-8053, 2008.

[29] H. Harald, N. Michael, W. Christoph et al., "Nanoscale morphology of conjugated polymer/fullerene based bulk heterojunction solar cells," Advanced Functional Materials, vol. 14, no. 10, pp. 1005-1011, 2004.

[30] L. Luo, Y. Zhang, S. S. Mao, and L. Lin, "Fabrication and characterization of $\mathrm{ZnO}$ nanowires based UV photodiodes," Sensors and Actuators, A, vol. 127, no. 2, pp. 201-206, 2006.

[31] G. Chai, O. Lupan, L. Chow, and H. Heinrich, "Crossed zinc oxide nanorods for ultraviolet radiation detection," Sensors and Actuators, A, vol. 150, no. 2, pp. 184-187, 2009.

[32] J.-S. Huang and C.-F. Lin, "Influences of $\mathrm{ZnO}$ sol-gel thin film characteristics on $\mathrm{ZnO}$ nanowire arrays prepared at low temperature using all solution-based processing," Journal of Applied Physics, vol. 103, no. 1, 2008. 

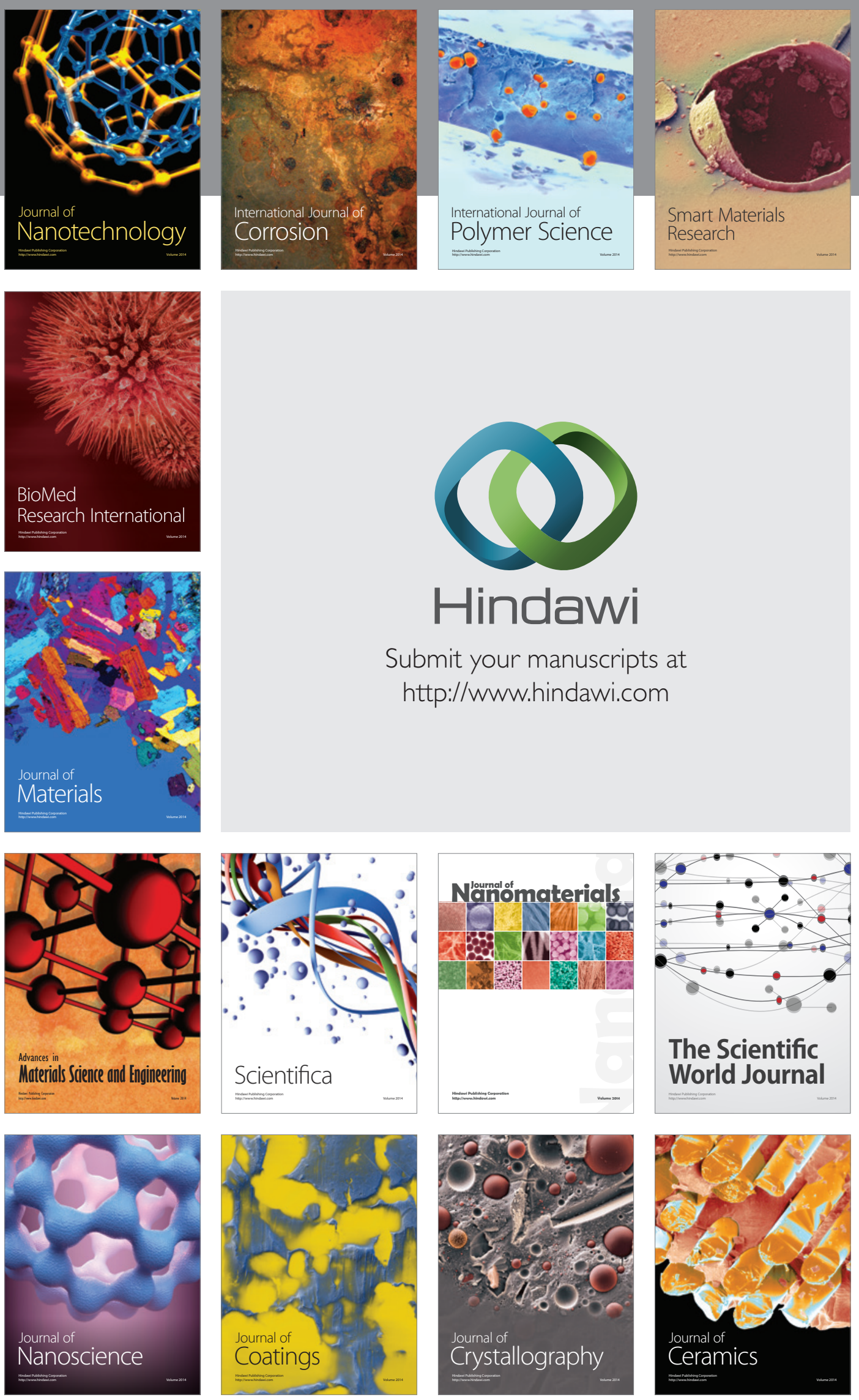

The Scientific World Journal

Submit your manuscripts at

http://www.hindawi.com

\section{World Journal}

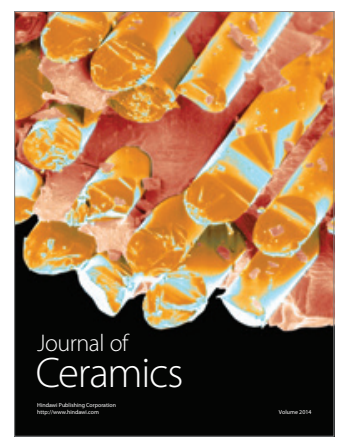

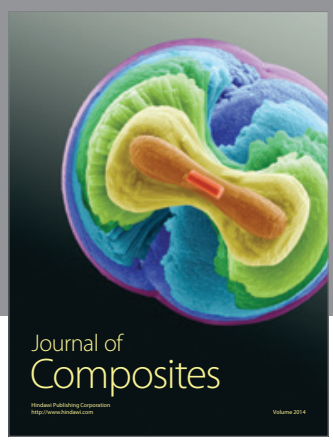
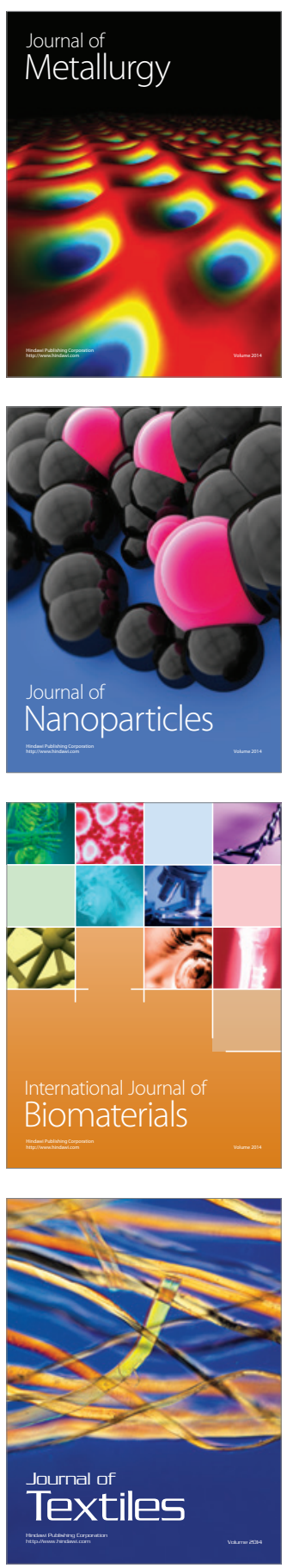